\title{
An unusual case of hypopituitarism and recurrent cranial nerve palsies
}

\section{BMAC Balasuriya, ${ }^{1}$ NP Somasundaram ${ }^{2}$ and $R$ Gamage $^{3}$}

(Index words: Intracerebral vasculitis, magnetic resonance angiogram)

We report here the history of a 52-year old woman with diabetes mellitus for 10 years and secondary hypothyroidism (low TSH) who presented with chronic persistent headache for 6 years and sudden onset of complete blindness of 24 hours duration, admitted to our unit at National Hospital of Sri Lanka in July 2004 . On the first admission in 1998, a cranial CT scan demonstrated a lesion suggestive of a macroadenoma of the pituitary gland, with posterior extension of the tumour towards the petrous temporal bone (Figure 1). The tumour was not

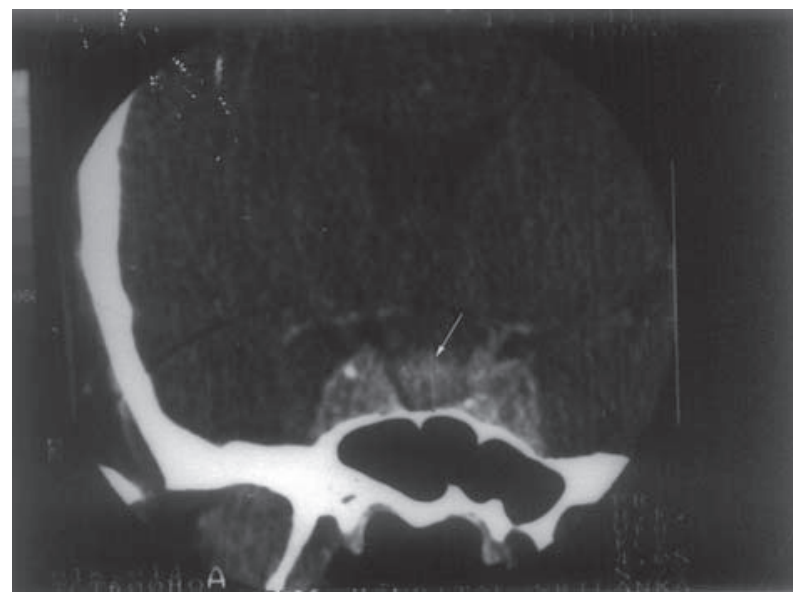

Figure 1. Macroadenoma of the pituitary (arrow).

removed as it was deemed unsuitable for surgical excision. She was started on medical therapy with bromocriptine, prednisolone, and thyroxine. A follow up CT scan in 1999 showed reduction in the size of the tumour height with no reduction in its extension to the cavernous sinus. MRI scan revealed thickening of the cavernous sinus and a compressed pituitary gland (54 mm). In the same year she was readmitted with a persistent headache and blurring of vision in the left eye.
Clinical examination at this stage revealed left-sided optic atrophy and bilateral abducens nerve palsy. In 2002, the next admission for a worsening headache revealed severe right-sided maxillary sinusitis. By this time her abducens nerve palsy was abated. She was treated with a course of co-amoxiclav and showed symptomatic improvement. The diabetes mellitus was under reasonable control with oral hypoglycaemic agents.

On clinical examination at the current admission, her BMI was 25 . She was pale with mild facial puffiness. Her pulse rate was $80 / \mathrm{min}$, and the $\mathrm{BP}$ was $100 / 80 \mathrm{mmHg}$. The cardiovascular and respiratory systems and the abdominal examination were clinically normal. Her higher cerebral functions, cerebellar functions, peripheral motor and sensory systems were intact. Cranial nerve examination showed bilateral dilated pupils, but there was no ophthalmoplegia. Funduscopy demonstrated bilateral optic atrophy. On the second day after admission, the patient developed right-sided oculomotor and abducens nerve palsies. She was treated with a large dose of prednisolone. At the end of 3 weeks of hospital stay her oculomotor and abducens nerve palsies had completely disappeared.

Laboratory investigations revealed haemoglobin of $10.4 \mathrm{~g} / \mathrm{dL}$, white blood cell count $14600 \mu / \mathrm{L}$ (neutrophils $70 \%$, lymphocytes $25 \%$ ), platelet count $20300 \mu / \mathrm{L}$, fasting blood glucose $5 \mathrm{mmo} / \mathrm{L}$, blood urea $3 \mathrm{mmol} / \mathrm{L}$, sodium $143 \mathrm{mmol} / \mathrm{L}$, potassium $3.8 \mathrm{mmol} / \mathrm{L}$. ESR was $70 \mathrm{~mm}$ in the first hour and serum calcium $2.18 \mathrm{mmol} / \mathrm{L}$. Antinuclear factor was negative, and antineutrophil cytoplasmic antibodies (PR3 ANCA) negative. Liver function tests were normal. The chest radiograph was normal. Blood picture revealed normocytic normochromic anaemia. The pituitary hormone estimations showed, serum basal 9 a.m. cortisol $36 \mathrm{nmol} / \mathrm{L}$, growth hormone $1 \mathrm{mU} / \mathrm{L}$ (normal range $<20 \mathrm{mU} / \mathrm{L}$ ) serum prolactin $1071 \mathrm{mU} / \mathrm{l}$ (normal range $0-400 \mathrm{mU} / \mathrm{L}$ ). $\mathrm{LH}<1 \mathrm{U} / \mathrm{l}$,

\footnotetext{
${ }^{1}$ Registrar in Medicine, ${ }^{2}$ Endocrinologist, ${ }^{3}$ Neurologist, National Hospital of Sri Lanka. Colombo, Sri Lanka. Correspondence: NP, e-mail: <noelps@hotmail.com> (Competing interests: none declared). Received 20 November 2004 and accepted 30 November 2004.
} 
FSH $<1 \mathrm{U} / \mathrm{l}$. The CSF analysis showed protein $44 \mathrm{~g} / \mathrm{dL}$, lymphocytes 4 , no red blood cells, glucose $81 \mathrm{mg} / \mathrm{dL}$.

Neuroimaging with MRI (gadolinium enhanced) showed a normal sized pituitary gland with uniform enhancement. The space occupying lesion seen in the previous scan had disappeared. The infundibular stalk could not be identified. However, the optic chiasm was widened ( Figure 2). The possibility of a granulomatous lesion was suggested by the radiologist. Intracerebral vasculitis was considered a possibility. Magnetic resonance angiogram of the cerebral vasculature revealed paucity of distal branches of the middle cerebral and both posterior cerebral arteries (Figure 3).

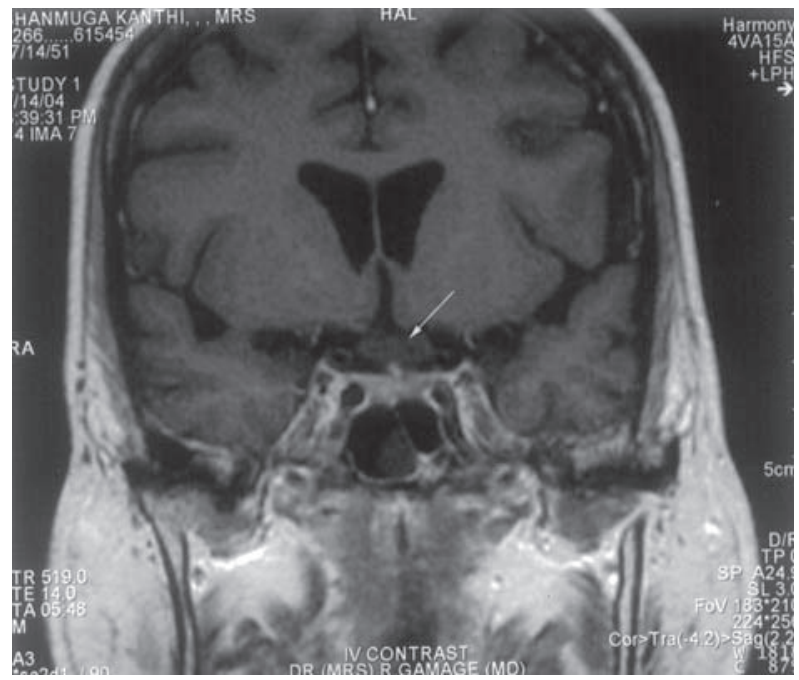

Figure 2. Widened optic chiasma (arrow).

\section{Discussion}

This patient presented with hypopituitarism, progressive optic atrophy and recurrent third and sixth cranial nerve palsies. The nerve palsies resolved each time probably as a result of the prednisolone treatment. She also had a lesion involving the pituitary gland, the left cavernous sinus, and the left side of the optic chiasm. The pituitary lesion seemed to resolve over the course of 5 years, leaving only a thickened cavernous sinus. When considering all the clinical features, there are a few diagnostic possibilities. A pituitary macroadenoma which has undergone massive necrosis is one of them. But there is no episode of pituitary apoplexy here. Furthermore, this diagnosis might not explain the intermittent nature of cranial nerve involvement. Diabetic mononeuritis could cause the individual nerve palsies. But the anatomical and chronological clustering is hard to explain on this basis alone. A granulomatous lesion of the pituitary region is another possibility, with a probable response to the steroid treatment. Finally, although the patient did not have systemic manifestations of autoimmune disease or vasculitis, as shown by the negative autoantibody screen, it is still a possibility. Isolated central nervous system

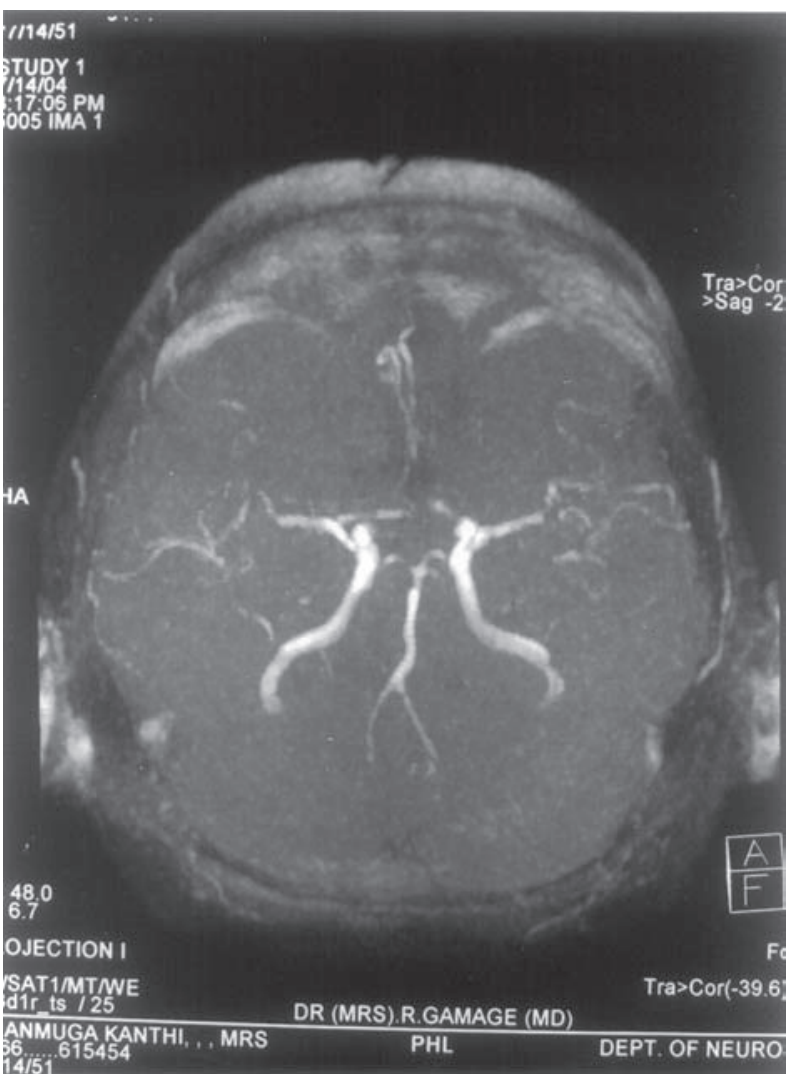

Figure 3. Magnetic resonance angiogram showing paucity of distal branches of the middle cerebral and both posterior cerebral arteries.

vasculitis (ICNSV) is an entity known to exist without evidence of systemic involvement, manifesting only as neurological dysfuction. It could present with persistent headache, personality changes, cranial neuropathies or weakness [1]. A fluctuating course of cranial nerve involvement favours diagnosis of intracerebral vasculitis, supported by the magnetic resonance angiographic evidence of missing vessels in two arterial territories in the circle of Willis. The usual features demonstrated in cerebral angiography in the case of ICNSV include beading of vessels, aneurysm formation, circumferential or eccentric vessel irregularities, multiple occlusions with sharp cutoffs and avascular mass effect. But none of these findings are diagnostic of ICNSV [2]. Interestingly, these patients may not show any vascular changes in the extracranial vessels, including temporal arteries. MRI scan may not show any specific brain parenchymal abnormality.

Granulomatous disease of the central nervous system could progress in a similar sequence to this case. There are few case reports of neurosarcoidosis presenting as cavernous sinus syndrome in the form of either the anterior, middle or the posterior syndromes [3]. In one of the series where the patient presented with right-sided ophthalmoplegia, all the preliminary investigations for sarcoid including the serum angiotensin converting enzyme levels and the CSF analysis were negative. Histopathological specimens showed the presence of 
granulomas with multinucleated giant cells [4]. It has been found that common laboratory investigations and CSF analysis are not sensitive enough to diagnose neurosarcoidosis. In our patient, histological examination of the lesion would have been useful in sorting out the current diagnostic dilemma. But it was not attempted on this admission, as there was no specific target for biopsy. Although the optic chiasm was widened, it was thought to be secondary to a fibrotic process.

The disease is now quiescent, except for a ESR of $70 \mathrm{~mm}$. She continues to be on replacement therapy. Should another flare-up occur, we hope to attempt a biopsy of the lesion to obtain a conclusive tissue diagnosis.

\section{References}

1. Calabrese LH, Duna GF, Lie JT. Vasculitis in the central nervous system. Arthritis and Rheumatism 1997; 40: 1189201.

2. Alhalabi M, Moore PM. Serial angiography in isolated angiitis of the central nervous system. Neurology 1994; 44: 1221-6.

3. Jefferson, N. Sarcoidosis of the nervous system. Brain: a Journal of Neurology 1957; 80: 540-57.

4. Zarei M, Anderson JR, Higgins JN, Manford MR. Cavernous sinus syndrome as the only manifestation of sarcoidosis. Journal of Postgraduate Medicine 2002; 48: 119-21.

\section{Cardiovascular mortality and morbidity from rofecoxib}

On 7 September this year, Merck Sharp \& Dohme faxed a "Dear Doctor" letter to Australian doctors to reassure them of the cardiovascular safety of rofecoxib. Yet, 3 weeks later, Merck Sharp \& Dohme suddenly announced an immediate worldwide withdrawal of rofecoxib; this action is the largest prescription drug withdrawal in history. In response, the United States Food and Drug Administrations (FDA) immediately issued a public health advisory on rofecoxib, while Australia's Therapeutic Goods Administration issued a customer-level recall for an estimated 250 000-300 000 Australians taking the drug.

PE Langton, GJ Hankey, JW Eikelboom. Medical Journal of Australia 2004; 181: 524-5 (Editorial) 